\title{
ON FORMAL TECHNIQUES IN PROTOCOL ENGINEERING - EXAMPLE CHALLENGES
}

\author{
Dines Bjørner \\ Informatics and Mathematical Modelling, Technical University of Denmark \\ Building 322, Richard Petersens Plads, DK-2800 Lyngby, Denmark \\ URI: www.imm.dtu.dk/ db, E-Mail: db@imm.dtu.dk
}

Abstract In this invited paper - for the 2001 FORTE Conference on FORmal Description TEchniques for Protocol Specification, Testing and Verification - we intend to illustrate a number of methodological issues that the practising software engineer might wish to consider when developing software for interacting, distributed software, more precisely, for such software which significantly contains interaction protocols. We "attach" our "guided tour" of software engineering using formal techniques, and following a triptych of domain engineering, requirements engineering, and software design, to two example "challenges". The particular examples are the domain and requirements for an "inverse" smart card application, that is, of a domain where ordinary citizens gather, refer to and update ("change") accumulated information and money resources and of requirements to a "really" smart card system which is the primary carrier of (ie. repository for) such information, and the (electronic) monies (themselves). The other example is that of "market" transactions; that is, a domain of interactions between government $(G)$ institutions, businesses $(B)$, and citizens $(C)$. That is with $G \leftrightarrow B \leftrightarrow C$ sequences of interactions - ie. "protocols", as well as some thoughts on requirements (an "extended E-Business system").

We outline the problems while adhering to our approaches to both informal and formal software engineering, to the development triptych, and indicate some of the very many principles and techniques inherent in any good method. By showing two different examples we illustrate the "impossibility" of one simpe, straightforward method.

Thus the paper presents a capsule view of some of our view of the Theory \& Practice of Software Engineering. ${ }^{1}$

${ }^{1}$ This is also the title of: [1]. 


\section{INTRODUCTION}

Protocol engineering, a sub-discipline of software engineering, as automotive engineering is of mechanical engineering, is a complex professional field. Protocol designers understood - earlier than software engineers in general - the importance of formal specification and verification.

Perhaps protocol engineers focused more on abstractly specifying intricate protocols and verifying some of their properties, than on developing, in general, provably correct software that satisfied the protocol specifications? This paper is about the latter.

In this presentation we wish to alert the field of protocol engineering to a number of more general software engineering development principles and techniques whose use in other fields of software engineering has been of some use.

Our two main points are those of the software engineer vigilantly following and adhering to a long check list of method principles and techniques, and that protocol development step back to include careful considerations of the actual world, ie. the domain, in which protocols serve, to better understand as many of the issues that ultimately lie behind a great number of protocol properties.

For software engineering to eventually label itself a professional engineering activity it must, it seems, rely, as do other engineering professions, on high specialisation - hence our insistence on referring to protocol engineering. Specialisation is characterised in terms of varieties of principles, techniques and tools whose conditional application follows careful analyses. Our field is slowly accumulating and enunciating these principles, techniques and tools. It may take a whole generation more to get them into general use.

\section{1. "Formal" Methods}

1.1.1 A Caveat. The label "formal" method' has been affixed to such techniques as use "formal" specification and "formal" verification. Here we first double quote the term 'method', secondly we "reduce" the scope of 'formal' from 'method', to 'specification \& ${ }^{2}$ verification'. Double-quoting expresses that we do not quite mean what we write: Methods cannot be "formal", but some of the techniques "they" imply may.

\footnotetext{
${ }^{2}$ The ' $\&$ ' connective is here used in its conventional meaning: Its two operands with ' $\&$ ' form one concept, not two !
} 
1.1.2 "What is a Method ?". Here the double quoted slanted display line title text designates a rhetorical question. Our answer is: A method is a set of analysis and synthesis principles for selecting and applying a number of techniques and tools in order efficiently to construct an efficient artifact. Here those artifacts are software.

Principles are to be adhered to, ie. followed, by humans. Hence they cannot be formalised, ie. are not "formal". Some techniques and tools (addressing such techniques) can be said to be formal. Specification languages are tools, and certainly can be formal: Have formal semantics, based on formal syntax, but never a formal pragmatics: Pragmatics is what leads outside formality: Has to do with the socio-economic context in which any utterance is made. Hence methods cannot be "formal", but crucial aspects of techniques and hence their associated tools can.

\subsection{A Triptych of Software Engineering}

Before software can be designed we must understand its requirements, hence they must (first ?) be engineered. Before requirements can be developed we must understand the (application) domain, hence it must (first ?) be engineered. Our approach is to both informally and formally describe all three: Domains, requirements and software - perhaps — in many stages and steps of "refinement". And to relate these phases, stages and steps.

\subsection{Problem Synopses}

Engineering is "walking the bridge between science and technology": "Constructing artifacts (here computing systems, notably software) based on scientific insight," and "investigating technology in order to ascertain its possible scientific value."

In this paper, as in so much else, we, engineers, are "driven" (forward, or is it sometimes "backwards") by the promises of the hard technologies.

In the case of this paper it is the "technologies" of "smart-cards" and "Internet", and their "derivatives" that first "drive' us.

1.3.1 The Citizen Smart Card: The Technology Incentive. Imagine, a few years from now, that low power, mass storage, fast computing \& optical communication is being facilitated by credit card sized (incl. thin) cards - which we shall henceforth refer to as The Citizen Smart Cards.

The Citizen Smart Card is capable, we conjecture, of holding, typically all of a citizens accumulated health-care information, and more, from a full lifetime: Information about all the health states, temperatures, 
blood pressure, blood sugar, cholesterol content, etc., all the original ECGs ever measured, all the original X-Rays ever taken, special photos taken before and after cosmetic (plastic) surgery, all the patient medical records ever recorded during hospital and clinic visits, your family doctors' notes about your health. All of this time stamped, etc.; all of a citizens accumulated financial information, again for any full lifetime, as well as all financial instruments currently holdable by any such citizen - ie. the monies themselves suitable transport timetables as well as commuter (daily, weekly, monthly or seasonal) tickets.

Add to the above that the first two kinds of information held on The Citizen Smart Card,in principle, is held nowhere else: No copies! ${ }^{3}$ The citizen is in sole and full possession of all own information!

The development brief now being posed, as our first example, is: Now what does the above imply in a world where we forget this card, where we loose it, where card terminals break down, etc.? Which citizen/card/machine interaction protocols does this call for?

We will try "edge" towards an answer by examining and modelling, also formally, the actual world of these three information source in the actual domain, and then suggest properties that a protocol and its supporting machinery required to satisfy.

1.3.2 E-Business: The Technology Incentive. The Internet is here. Here and there, contractual transactions take place over the net. Merchandise (ie manifest, tangible goods, instantly tradeable, transferable) and (intangible, time-interval providable) services are offered, are sold, are sought, are bought, etc.

So far two categories of traders: Buyers and sellers have been identified: Businesses $(B)$ and citizens (or consumers, $C$ ). But in general there are three, the above and government institutions $(G)$.

The question is now: How to generalise the notions of $B 2 C$ and $B 2 B$ transactions to arbitrary pairings of traders (buyers and sellers) from the three categories; and how to extend these transactions to such which involve "middlemen": Agents and brokers?

The development brief now being posed, as our second example, is in the domain — to investigate the full triangle of pairwise interactions; identify "primitive" such transactions, such which enter into most transaction sequences; explore possible behaviours; and - as requirements — to identify suitable protocols.

${ }^{3}$ That is: No copies, in a logical sense. Physically, instead of The Citizen Smart Card storing bulks of information it may instead refer to more stationary repositories where (sole) originals are kept. Still, the idea is that the citizen is in full control. 


\subsection{Structure of Paper}

In Section 2 we informally and formally describe the two domains of the citizen information "repository", and "the market". Based on that, we can, in Section 3, outline The Citizen Smart Card and E-Business. requirements - emphasising their protocol aspects. In those sections, and in Section 4, we review a number of method principles and techniques. Space limitations do not permit a thorough treatment - so we shall refer, instead, to published papers and a forthcoming text book on methodical software engineering based on the use of formal techniques.

\section{DOMAIN ENGINEERING}

Domain engineering is not the same as knowledge engineering. The latter could be said to be "a subset of" the former. In knowledge engineering, briefly, computing scientists and knowledge engineers try model the knowledge of stake-holders of the domain in such a way that the knowledge models are "directly" computable. In domain engineering we do not consider computable aspects only. We wish to model as much of the domain that can be described. We refer to [2] for details.

\subsection{The Citizen Smart Card: A Domain}

2.1.1 Three Sub-Domains: Informal Sketches. We treat three diverse information and resource "gathering" and "dispensing" domains: citizen health information, financial information and securities, and transport services and tickets.

Patient Medical Records (PMRs) and Journals (PMJs) By a Patient Medical Record, PMR, we mean a collection of documents pertaining to one particular health case of one particular citizen - whether that citizen sought help from medical professionals or not. At any one time several PMRs may be in effect: The citizen may be analysed or undergoing treatments for more than one disease or similar. By a Patient Medical Journal, PMJ, we mean the collection of all PMRs, over the entire life-time of that patient, "up till now!".

We refer to PMRs, not necessarily as manifest, tangible paper, instrument output strips or X-ray files, documents, but, in addition to such, also as what the citizens (the patient, relatives working place colleagues), in the past remembered, or now remembers. We certainly do not have electronic PMRs in mind but do not a-priori rule them out either. 
In other words: A PMJ represents all that is knowable, by whatever means, about a citizen's health.

A PMJ may be subject to a number of operations: (1) creation, ie. opening of new PMRs, and (2) closing, ie. "filing away" of existing PMRs. Individual PMRs may be subject to a number of operations: (3) entering and update of administrative information: Patient name, dates, addresses, etc.; (4) entering and update, incl. "deletion" of "raw", undigested (ie., further unanalysed) medical information obtained from the patient, "previous" PMRs, relatives, etc., or obtained as analysis results from medical professionals (or other), or in the form of analysis originals, or from, analytical (medico-technical) instruments (ECGs, blood analysis, X-Rays, etc.); (5) entering and update, incl. "deletion" of diagnostics; (6) entering and update of treatment plans; (7) entering and update of treatment actions; (8) entering and update of how the patient responds to treatment actions. Whereby we are back to item (4).

In addition we can inquire about PMR/PMJ information: search, correlate, and compute over such information - as do medical professionals: When analysing, when diagnosing, etc.

That's basically "all!"

We refer to two kinds of operations and of two kinds of queries: those which update PMJs with no input provided (O), those which update PMJs with input provided (IO), those which query PMJs with no input provided $(Q)$, and those which update PMJs with input provided (IQ).

Financial Services We treat only banking and securities trading.

- Banking Today you would be expected to have a number of bank accounts (Acc): Savings \& Loan (S\&L), Demand/Deposit, Cheque, etc. Each of these show a balance, either positive or negative. Each such balance represents credit or debit in the bank. One may perform the following operations related to accounts: open, close (new, resp. old account), save, borrow, repay (in preparation for loan, establish loan, repay loan - in installments), withdraw, deposit, change credit (latter stands for extending or lowering credit limit), statement (list of transactions "since last"), etc.

The development brief calls for an analysis of the bank/customer interaction interface today - with a view towards keeping the banks, but maintaining all information and all balances (whether negative or positive) - that is: "the real monies" — on The Citizen Smart Card in future. 
- Securities Trading Securities trading is an important financial service: Enterprises and governments offer securities (stocks, respectively bonds, Sec), for sale. Enterprises and people buy and sell stocks and bonds. Stocks and bonds are traded on the "floor" of stock exchanges. Brokers and traders act as middlemen between buyers and sellers. The following are typical securities trading operations: initial stock offering; placing and withdrawing orders to buy, respectively sell; effect, as it were "simultaneously" a batch of one or more buy/sell orders; transfer of securities instruments: The stock, bonds and payments. Ec.

The development brief calls for an analysis of the client/broker interaction interface today - with a view towards keeping the broker and securities exchanges, but maintaining all information and all securities instruments (stocks, bonds) on The Citizen Smart Card in future.

Public Transport We shall look at Public Transport only from the very narrow perspective of potential passengers (incl. commuters). Commuters possess knowledge of time and fare tables. Commuters enter, pay for (cash or by showing seasonal tickets), and leave transport vehicles (taxis, busses, street cars, metro trains, canal boats, etc.).

The development brief calls for an analysis of the commuter/transport system interaction interface today - with a view towards keeping the transport, but maintaining all information (time and fare tables), and all monies and tickets (InfoTick) on The Citizen Smart Card in future.

2.1.2 Analysis and Formal Model. We decompose our analysis and models into data (ie. type) and function (incl. process) models.

Data Analysis The unifying quantity, whether PMR, PMJ, Sec, or Info Tick, will be referred to as R. Operations on (ie. functions that change) PMR, PMJ, Sec, or InfoTick, will be referred to a O. Queries on PMR, PMJ, Sec, or InfoTick, will be referred to a $Q$. Extensions of these are operations or queries which update with special information or query wrt. such information $\mathrm{I}$.

\section{Formal Domain Data Model}

$$
\begin{aligned}
& \text { type } \\
& \mathrm{R}, \mathrm{I} \\
& \mathrm{O}=\mathrm{R} \rightarrow \mathrm{R} \\
& \mathrm{Q}=\mathrm{R} \rightarrow \mathrm{I} \\
& \mathrm{IO}=\mathrm{I} \times \mathrm{R} \rightarrow \mathrm{R} \\
& \mathrm{IQ}=\mathrm{I} \times \mathrm{R} \rightarrow \mathrm{I}
\end{aligned}
$$


Process Analysis Whether a PMR or a PMJ: moving around the health-care system, being tested, taking medicine, having a fever, etc.; or a bank account or a securities instrument (Acc, Sec): depositing and withdrawing cash into and from an account (etc.), buying or selling stocks and bonds (etc.), etc.; or a transport repository (Info Tick): obtaining new time table and fare information, boarding and leaving a bus (train, etc.); all amounts to updating or querying the repository.

Formal Process Model Each repository is like a process. Each such process is, non-deterministically, subject to any of a great variety of operations or queries.

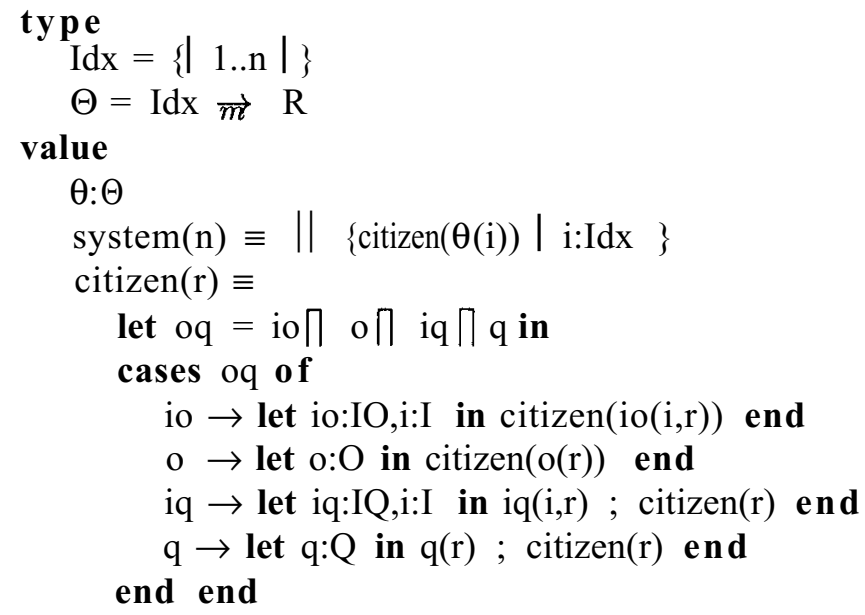

The system of all $(n)$ citizens proceed, in the domain, as a set of noninterfering parallel processes. Each citizen non-deterministically (internal choice, П.) perform an information-based update (io), a "standard" (a simple) update (o), an information-based query (iq), or a (simple) query (q).

To paraphrase: That's all !

Discussion We remind the reader that the narration and formalisation above is of a domain, not of requirements to software. And we remind the reader that we are focusing on the repository of information and certain (here the financial) resources.

In this example the formal domain model is very trivial. It expresses only the very basics. In the domain all is possible: Humans err, are not consistent, etc. One reason for seeking computing and communication support is to help avoid mistakes and to secure consistency. 
The protocol issue was highlighted: We have modelled the non-deterministic handling, ie. arbitrary interleaving of the operations and queries of this information and resource repository. We have abstracted which these operations and queries were in specific.

The next example will be in contrast to the present.

\subsection{E-Business: A Domain}

2.2.1 Government, Business and Citizen Transactions. In the case of the government, business and citizen infrastructure interactions we can postulate the following domain, ie. not necessarily computer $\&$ electronic communication supported, transactions:

$G 2 G$ : Government institutions, $G$, buy services from other $G$, and $G$ sell services to other $G$. The $G$ pay with monies (obtained through taxes, etc.), respectively offer free services, in return. $G 2 B: G$ buy services, or request taxes, from businesses $(B)$, and pay, respectively offer free services, in return. $G 2 C$ : $G$ buy services (hire), or request taxes, from citizens $(C)$, and pay, respectively offer free services, in return. $B 2 G$ : Businesses $(B)$ buy services from $G$, and pay $B$ for these either through having already paid taxes or by paying special fees. $B 2 B: B$ buy merchandise or services from other $B$, and $B$ offer merchandise or services to other $B$. $B$ usually pay for these outright. $B 2 C: B$ buy services from citizens: ie. hire temporary or permanent staff (employment), and $B$ pay for these through salaries. $C 2 G$ : Citizens $(C)$ obtain services from $G$ (passport, drivers licence, etc., health-care, education, safety and security, etc.) and $C$ pay for these either by paying special fees or through having already paid taxes. $C 2 B: C$ buy merchandise from $B$, and $C$ pay for this. $C 2 C$ : Two or more $C$ together enter into political "grass-root" organisations, or leisure-time hobby club activities, or just plainly arrange meetings (incl. BBQ parties); and the two or more $C$ "pay" for this by being "together".

2.2.2 Traders: Buyers and Sellers. Above we have stressed that also government (institutions) are part of the more general concept of E-Business, some aspects of contractual obligations, and a seeming "symmetry" between partners to any such contract (ie. buy, sell, etc.). As such we have stressed that "The Market" consists of buyers and sellers, whom we, as one, refer to as traders.

2.2.3 Traders: Agents and Brokers. An agent, to us, while we are still only discussing the domain, is a trader that acts (in a biased manner) on behalf of usually one other trader (either a buyer, or a seller), vis-a-vis a number of other traders (sellers, respectively buyers), in 
order to secure a "best deal". A broker, to us, while we are still only discussing the domain, is a trader that acts (in a neutral manner) on behalf one or more buyers and one or more sellers in order to help them negotiate a "deal."

2.2.4 Schematic Transactions. Sequences of contractual transactions can be understood in terms of "primitive" transactions:

A buyer inquires as to some merchandise or service. A seller may respond with a quote. A buyer may order some merchandise or service. A seller may confirm an order. A seller may deliver an order. A buyer may accept a delivery. A seller may send an invoice. A buyer may pay according to the invoice. A buyer may return, within warranty period, a delivery. And a seller may refund such a return.

We have, deliberately, used the "hedge" 'may':

A trader may choose an action of no response, or a trader may inform that a transaction was misdirected, or a trader may decline to quote, order, confirm, deliver, accept, pay orrefund!

\subsubsection{Formalisation of Syntax.}

$$
\begin{aligned}
\underset{\text { Trans }==}{\text { type }} & \text { Inq } \mid \text { Ord } \mid \text { Acc } \mid \text { Pay } \mid \text { Rej } \\
& \text { Qou } \mid \text { Con } \mid \text { Del } \mid \text { Acc } \mid \text { Inv } \mid \text { Ref } \\
& \text { NoR } \mid \text { Dec } \mid \text { Mis }
\end{aligned}
$$

The first two lines list the 'buyer', respectively the 'seller' initiated transaction types. The third line lists common transaction types.

$\mathrm{U}$ below stand for unique identifications, including time stamps $(T)$, Sui for surrogate information, and MQP alludes to merchandise identification, quantity, price.

$$
\begin{aligned}
& \mathrm{U}, \mathrm{T}, \mathrm{Su} 1, \mathrm{Su} 2, \mathrm{MQP} \\
& \mathrm{Inq}=\mathrm{MQP} \times \mathrm{U} \\
& \text { Qou }=(\mathrm{Inq} \mid \mathrm{Su} 1) \times \mathrm{Inf} \times \mathrm{U} \\
& \text { Ord }=\text { Qou } \mid \mathrm{Su} 2 \times \mathrm{U} \\
& \mathrm{Con}=\text { Ord } \times \mathrm{U} \\
& \text { Del }=\text { Ord } \times \mathrm{U} \\
& \text { Acc }=\text { Del } \times \mathrm{U} \\
& \mathrm{Inv}=\text { Del } \times \mathrm{U} \\
& \text { Pay }=\text { Inv } \times \mathrm{U} \\
& \text { Rej }=\text { Del } \times \mathrm{U} \\
& \text { Ref }=\text { Pay } \times \mathrm{U} \\
& \text { NoR }=\text { Trans } \times \mathrm{U} \\
& \text { Dec }=\text { Trans } \times \mathrm{U}
\end{aligned}
$$




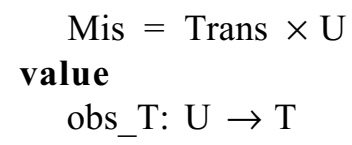

In general we model, in the domain, a "subsequent" transaction by referring to a complete trace. of unique, time stamped transactions. Thus, in general, a transaction "embodies" the transaction it is a manifest response to, and time of response.

Figure 1 attempts to illustrate possible transaction transitions between buyers and sellers.

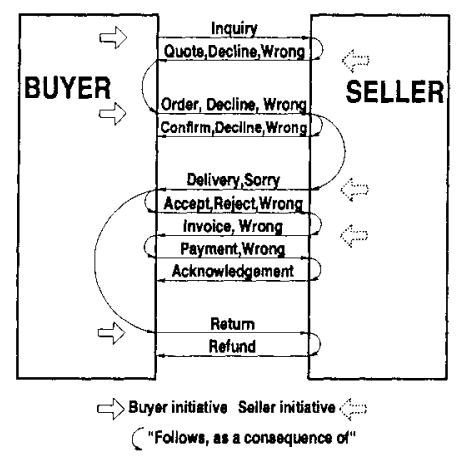

Figure 1. Buyer / Seller Protocol

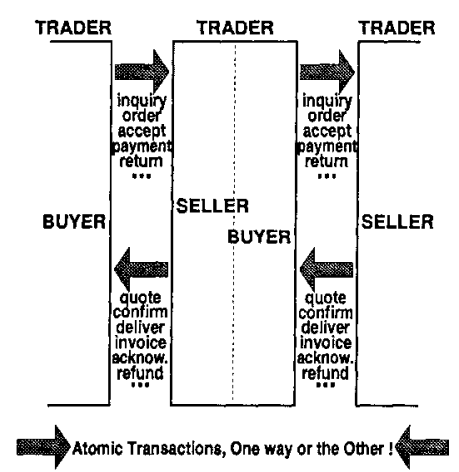

Figure 2. Trader $=$ Buyer + Seller

2.2.6 Transaction Sequences. Figure 3 attempts to show the possible sequences of transactions as related to one "trade": From inquiry through refunding, that is: For one pair of buyer/seller.

2.2.7 "The Market". Figure 2 attempts to show that a trader can be both a buyer and a seller. Thus traders "alternate" between buying and selling, that is: Between performing 'buy' and performing 'sell' transactions.

Figure 4 attempts to show "an arbitrary" constellation of buyer and seller traders. It highlights three supply chains. Each chain, in this example, consists, in this example, of a "consumer", a retailer, a wholesaler, and a producer.

2.2.8 Formalisation of Process Protocols. "The Market" consist of $n$ traders, whether buyers, or sellers, or both; whether additionally agents or brokers. Each trader $\tau_{i}$ is able, potentially to com- 


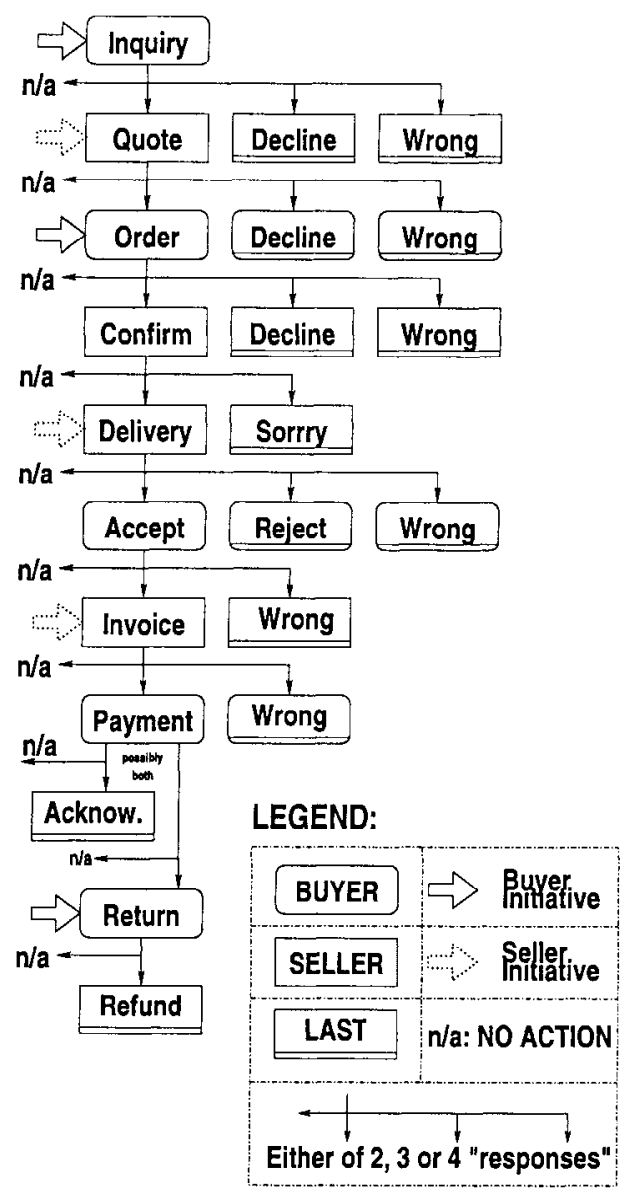

Figure 3. Transaction Sequence "Chart"

municate with any other trader $\left\{\tau_{1}, \ldots, \tau_{i-1}, \tau_{i+1}, \ldots, \tau_{n}\right\}$. We omit treatment of how traders come to know of one another. And focus only on the internal and external non-determinism which is always there, in the domain, when transactions are selected, sent and received.

Our model is in a variant of CSP, but expressed "within" RSL [3].

type

$\Theta$

$\operatorname{Idx}=\{|1 . . n|\}$

value 


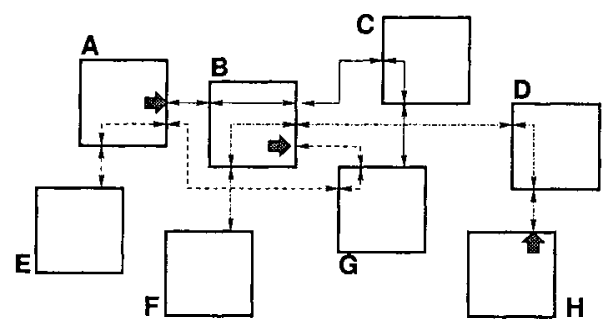

(Example Supply Chains: ABCG, HDBF, BGAE, ...)

Figure. 4. A Network of Traders and Supply Chains

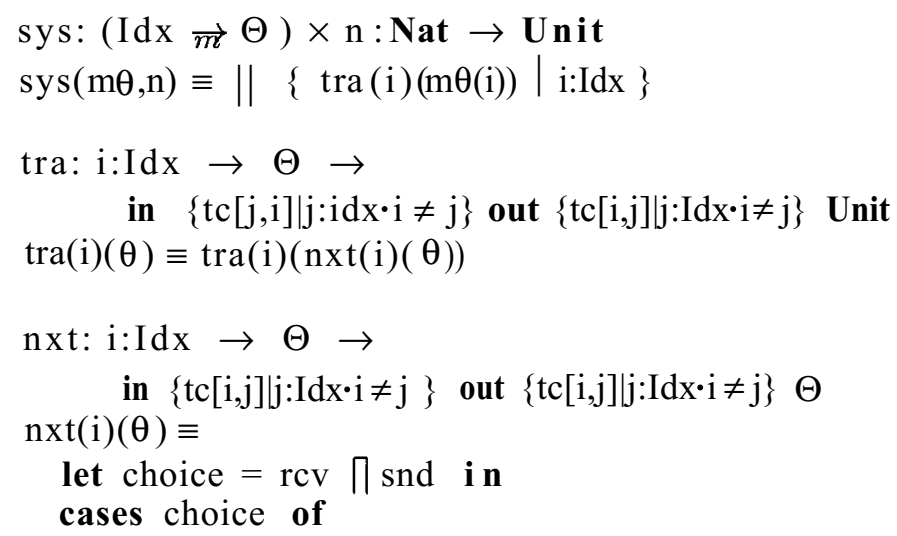$$
\operatorname{rcv} \rightarrow \operatorname{receive}(i)(\theta) \text {, snd } \rightarrow \operatorname{send}(i)(\theta) \text { end end }
$$

The system is the parallel combination of $n$ traders. Traders communicate over channels: $t c[i, j]$ - from trader $i$ to trader $j$. Each trader is modelled as a process which "goes on forever", but in steps of next state transitions. The next state transition non-deterministically (internal choice, П) "alternates" between expressing willingness to receive, respectively desire to send. In "real life", ie. in the domain, the choice as to which transactions are taken is non-deterministic. And it is an internal choice. That is: The choice is not influenced by the environment.

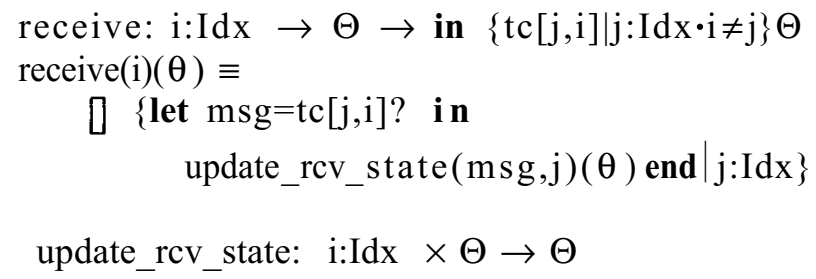


update_rcv_state is not a protocol function. update_rcv_state describes the deposit of $m s g$ in a repository of received messages. If $m s g$ is a response to an earlier sent transaction, msg-o, then update_rcv_state describes the removal of msg-o from a repository of sent messages. remove_set_msg models the situation where no response (nor) is (ever) made to an earlier sent message. Once the internal non-deterministic choice $\left(\prod\right)$ has been made: Whether to receive or send, the choice as to whom to 'receive from' is also non-deterministic, but now external (]). That is: receive expresses willingness to receive from any other trader. But just one. As long as no other trader $j$ does not send anything to trader $i$ that trader $i$ just "sits" there, "waiting" - potentially forever. This is indeed a model of the real world, the domain. A subsequent requirement may therefore, naturally, be to provide some form of time out. A re-specification of receive with time out is a correct implementation of the above.

send: $\mathrm{i}: \operatorname{Idx} \rightarrow \Theta \rightarrow$ in $\{\mathrm{tc}[\mathrm{i}, \mathrm{j}] \mid \mathrm{j}: \operatorname{Idx} \bullet \mathrm{i} \neq \mathrm{j}\} \quad \Theta$

send (i) $(\theta) \equiv$

let choice $=$ ini $\Pi$ res $\prod$ nor in

cases choice of

$$
\begin{aligned}
& \text { ini } \rightarrow \text { send_initial (i) }(\theta) \text {, } \\
& \text { res } \rightarrow \text { send_response (i) }(\theta), \\
& \text { nor } \rightarrow \text { remove_received_msg }(\theta) \text { end end }
\end{aligned}
$$

Either a trader, when communicating a transaction chooses an initial (ini) one, or chooses one which is in response (res) to a message received earlier, or chooses to not respond (nor) to such an earlier message The choice is again non-deterministic internal. In the last case the state is updated by non-deterministically internal choice (not shown) removing the, or an earlier received message.

Note that the above functions describe the internal as well as the external non-determinism of protocols. We omit the detailed description of those functions which can be claimed to not be proper protocol description functions - but are functions which describe more the particular domain at hand: Here "The Market".

$$
\begin{aligned}
& \text { send_initial: } i: \operatorname{Idx} \rightarrow \Theta \rightarrow \text { out }\{\operatorname{tc}[i, j] \mid j: \operatorname{Idx} \bullet i \neq j\} \Theta \\
& \text { send_initial }(i)(\theta) \equiv \\
& \text { let choice }=\text { buy } \Pi \text { sell in } \\
& \text { cases choice of } \\
& \quad \text { buy } \rightarrow \text { send_int_buy }(i)(\theta), \\
& \quad \text { send } \rightarrow \text { send_init_sell }(i)(\theta) \text { end end }
\end{aligned}
$$


send_response: $\mathrm{i}: \mathrm{Idx} \rightarrow \Theta \rightarrow$ out $\{\mathrm{tc}[\mathrm{i}, \mathrm{j}] \mid \mathrm{j}: \mathrm{Idx} \bullet \mathrm{i} \neq \mathrm{j}\} \Theta$

send_response $(i)(\theta) \equiv$

let choice $=$ buy $\Pi$ sell in

cases choice of

$$
\begin{aligned}
& \text { buy } \rightarrow \text { send_res_buy }(i)(\theta), \\
& \text { sell } \rightarrow \text { send_res_sell(i) }(\theta) \text { end end }
\end{aligned}
$$

In the above functions we have, perhaps arbitrarily chosen, to distinguish between buy and sell transactions. Both send_initial and send_response functions - as well as the four auxiliary functions they invoke - describe aspects of the protocol.

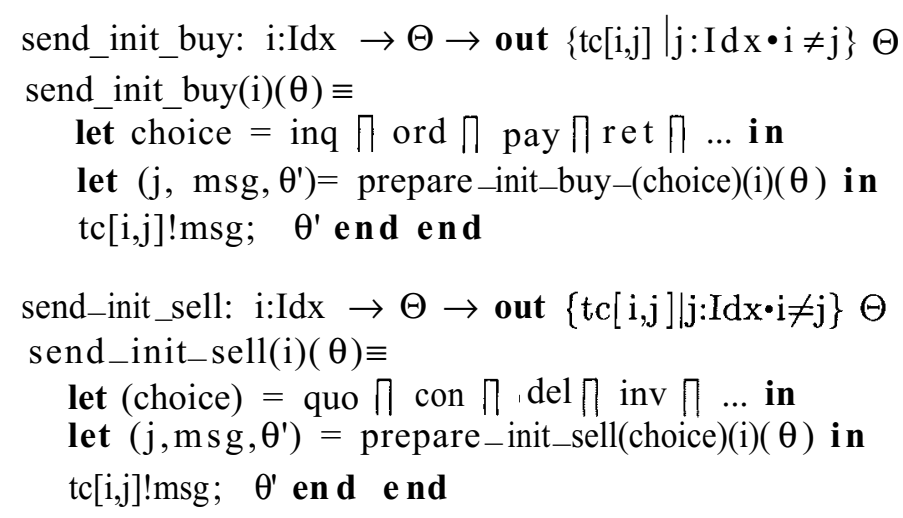

prepare_init_buy is not a protocol function, nor is prepare_init_sell. They both assemble an initial buy, respectively sell message, msg, a target trader, $j$, and update a send repository state component.

$$
\begin{aligned}
& \text { send_res_buy: } \mathrm{i}: \operatorname{Idx} \rightarrow \Theta \rightarrow \text { out }\{\mathrm{tc}[\mathrm{i}, \mathrm{j}] \mid \mathrm{j}: \operatorname{Idx} \bullet \mathrm{i} \neq \mathrm{j}\} \Theta \\
& \text { send_res_buy }(\mathrm{i})(\theta) \equiv \\
& \text { let }\left(\theta^{\prime}, \mathrm{msg}\right)=\text { sel_update_buy_state }(\theta) \text {, } \\
& \mathrm{j}=\text { obs-trader }(\mathrm{msg}) \text { in }
\end{aligned}
$$

$$
\begin{aligned}
& \text { send_res_sell: } \mathrm{i}: \mathrm{Idx} \rightarrow \Theta \rightarrow \text { out }\{\mathrm{tc}[\mathrm{i}, \mathrm{j}] \mid \mathrm{j}: \operatorname{Idx} \bullet \mathrm{i} \neq \mathrm{j}\} \Theta \\
& \text { send_res_sell }(\mathrm{i})(\theta) \equiv \\
& \text { let }\left(\theta^{\prime}, \mathrm{msg}\right)=\text { sel_update_sell_state }\left(\theta^{\prime}\right) \text {, } \\
& \mathrm{j}=\text { obs_trader(msg) in } \\
& \text { let }\left(\theta^{\prime \prime}, \mathrm{msg}^{\prime}\right)=\text { response_sell_msg }(\mathrm{msg})\left(\theta^{\prime}\right) \text { in } \\
& \text { tc }[i, j] \text { !msg'; } \theta \text { " end end }
\end{aligned}
$$

sel_update_buy_state is not a protocol function, neither is sel_update_sell_state. They both describe the selection of a previously deposited, 
buy, respectively a sell message, $m s g$, (from it) the index, $j$, of the trader originating that message, and describes the update of a received messages repository state component. response_sell_msg and response_buy_msg both effect the assembly, from $m s g$, of suitable response messages, msg'. As such they are partly protocol functions. Thus, if msg was an inquiry then msg' may be either a quote, decline, or a misdirected transaction message. Etcetera.

2.2.9 Discussion. In this example, in contrast to the E-Business example, the protocol aspect was quite "pronounced". Again we remind the reader that we have, so far only described facets of the domain. Next we shall deal with requirements.

\subsection{Discussion: Domain Engineering}

The two example cases differ widely: The Citizen Smart Card "resulted" in a "small" domain model. The E-Business model, in contrast, was quite substantive. The latter included quite an elaborate, yet, in essence "free" protocol: Any sequence of transactions will occur in the domain, and there were many variants. The former seems, in the domain, to have an utterly uninteresting "protocol" - if such a term could indeed be used!

\section{REQUIREMENTS ENGINEERING}

We illustrate a novel technique of "deriving" significant features of requirements from the domain description. The idea is that the domain descriptions (and ours were necessarily rather cursory) "point" to the necessity of certain domain requirements. Interface and machine requirements will only be cursorily covered. We refer to $[4,5,6]$ and the forthcoming [1] for details.

\subsection{Domain Requirements}

Domain requirements "construction" proceeds, it is suggested, by deploying the following techniques: Projection - where only those parts of the domain model which is of interest are "carried" forward to requirements. Instantiation - where abstract types and functions defined only through their signatures are made more specific. Extension - where new domain possibilities, afforded through computing and communication, but hitherto not feasible without, are "added" to the requirements (although "really" a domain issue). Initialisation - where the whole issue of how to initialise the context and state configuration components 
is dealt with. Ie., the input, vetting and update of configuration data. We shall mostly be concerned with projection and instantiation.

\subsubsection{The Citizen Smart Card.}

Projection Synopsis We project information and securities (incl. money) resources onto The Citizen Smart Card as mentioned earlier. Figure 5 attempts to show one possible rendition of The Citizen Smart Card. The Citizen Smart Card is to store all information and be an electronic purse.

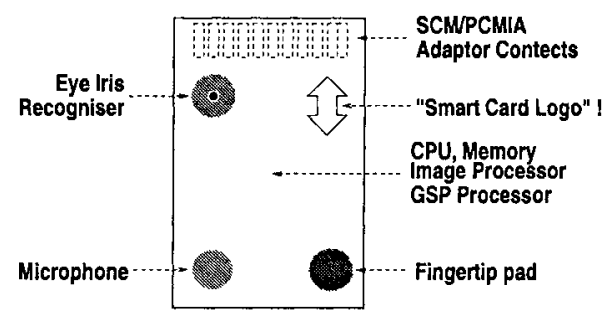

Figure 5. A Smart Card

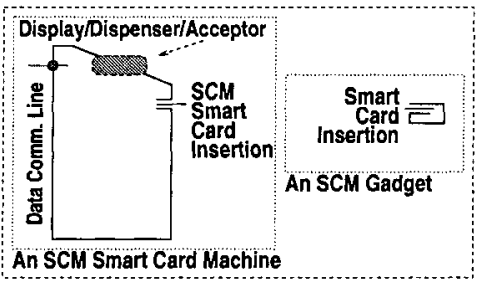

Figure 6. Smart Card Terminals

Instantiation Synopsis We omit consideration of how the repository information and electronic (purse) securities, ie. R, are represented.

Extension Synopsis Here is where some considerations of machine requirements (notably dependability issues) are to be made first. We refer forward to the machine requirements for the The Citizen Smart Card.

We extend as now outlined: To complement The Citizen Smart Card there must be means of "feeding" (health-care, financial and transport services) information onto the card and "moving" electronic money to and from the card. Figure 6 attempts to show two possible such means: An ETM/ATM-like machine, as shown to the left, and a "gadget" as indicated to the right. The ETM/ATM-like machines are well-known. The "gadgets" provide the means of writing and reading information to screens and from for example medico-electronics instruments, and bus and train ticketing gadgets.

Over time the citizen applies the card to a variety of such machines and gadgets (ie. terminals, SCM). Figure 7 attempts to show, as temporally determined, such a sequence of terminals: The Citizen Smart Card was first issued by SCMa, and last used in connection with SCMn. 
In-between The Citizen Smart Card was "applied" to SCMb, ..., SCMi, SCMj, SCMk, ..., and SCMm.

Ignore, for the moment, the arrows between SCMs .

But observe that the trace of SCM usage portrays a virtual, a logic usage: Two or more SCMx and SCMy (where $\mathrm{x}$ and $\mathrm{y}$ in a sense stands for temporal order), may be the same physical terminal. Thus there may be loops. In fact: They all may be the same terminal. The card holder is "provincial", never gets around!

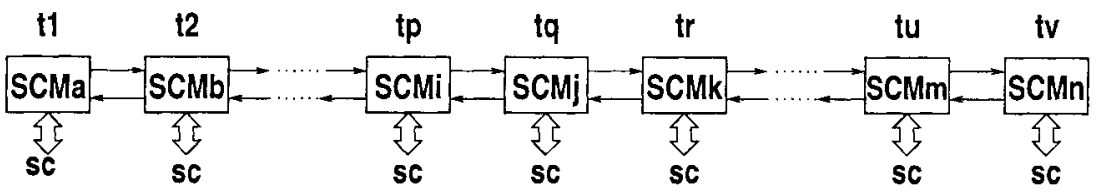

Figure 7. Trace of Terminal Use

Failures of Operation We can now discuss some possibilities: (i) The Citizen Smart Card may be lost. (ii) The Citizen Smart Card may be stolen. (iii) The smart card terminals may break down. ES.C.

We discuss possible protocols to be observed for each of these three situations.

(i) The Citizen Smart Card may be lost. We assume each card to have a unique identifier. We assume each SCM to remember all the uniquely time-stamped and card identified transactions it has been engaged in for all applied cards. By, for example, letting The Citizen Smart Card remember the identity of the SCM it was last applied to, a link can be made from the SCM it is currently applied to to that "previous" SCM, which, in turn, can then provide a link to the "current" SCM. If the holder of the The Citizen Smart Card can remember just one of the "past" SCMs, then a new card can be issued with all the accumulated and processed information and securities instruments as per the day of loosing the card. (ii) The Citizen Smart Card may be stolen. By providing, as indicated by Figure 5, a triple identification means: Eye iris "stamp", finger print, and voice print, any high degree of privacy can be obtained. A stolen card otherwise leads to the same re-establishment protocol as a lost card. (iii) The smart card terminals may break down. Figure 8 attempts to show a failing terminal SCMj. By further providing "leap frog" links, forward and backward, skipping one, as shown, two and/or more SCMs, together with copying also "neighbouring" transactions, a suitable redundancies can, again, help ensure recovery of data. And so forth. 
Now recall that the trace of terminals used by a card may use the same SCM repeatedly, or always! This could call, in general, for a set of SCM "buddies": SCMs that are physically different from the ("master") SCM of which they are "buddies". Now, instead of inserting links and copying transaction data to "master" SCMs - when the same as "current" SCM - they are copied, for example, to a rotating selection of "buddies". And so forth!

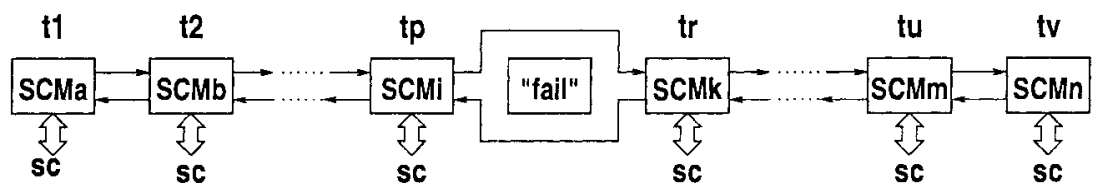

Figure 8. "Broken" Trace of Terminal use

Formalisation of Card/Terminal Interaction We leave out a formal model of the interlocking process of a card, mutually exclusively, interacting with a terminal.

\subsubsection{E-Business.}

Projection Synopsis We omit consideration of all operations and queries, ie. of any specific 0 , io, $q$ and iq in respectively $O, I O, Q$ and IQ. and focus only on the communication between traders. We basically ignore the "content" of any transaction, and shall instead focus on automating certain sequences of transactions.

Instantiation Synopsis Whereas the domain model of traders was a model, essentially, intrinsically, of human operations, we now try to automate as much as possible the response to received transactions. Thus, as an example: (1) If a consumer order can be delivered by the retailer, without human (retailer staff) intervention, it will be done so. (2) If a consumer order cannot be delivered by the retailer, but that retailer can re-order from a wholesaler, who can deliver — both atomic transactions without human (retailer and wholesaler staff) intervention, it will be done so. (3) And if a consumer order cannot be delivered by the retailer, but that retailer can re-order from a wholesaler, who then cannot deliver, but must re-order from producer, who can deliver - all atomic transactions without human (retailer, wholesaler and producer staff) intervention, it will be done so. 
Figure 9 attempts to show the three cases listed above. There might be delays, waiting times, between order receipt and delivery and/or reordering, across the supply-chain.

Extension Synopsis We introduce electronic traders and brokers. They permit arbitrarily wide inquiries: Potentially to all providers (retailers, wholesalers, or producers) of specified merchandise (or services), offers ("confirmations") of merchandise (or services) to all "takers" (consumers, retailers, or wholesalers), first-come-first serve ("auction"-like) orders, $\mathscr{E} c$. These roughly sketched domain requirements are considered extensions as they might not be humanly feasible in actual domains.

Three Supply-chain Cases: (c1) Direct, (c2) via Retailer, (c3) all the way from Producer

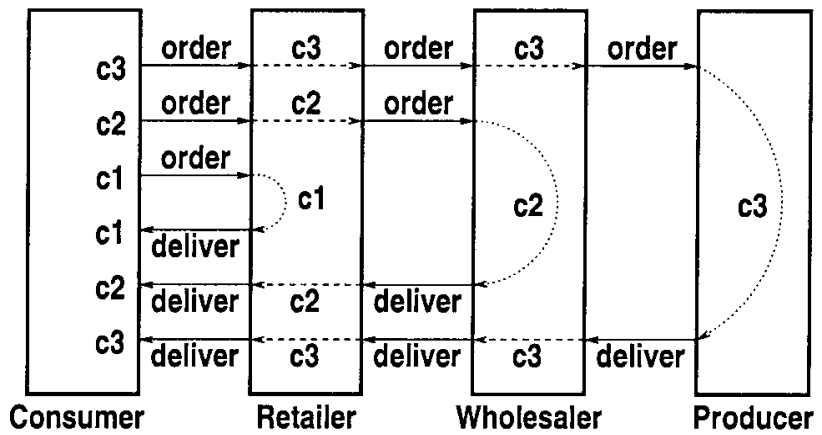

Figure 9. E-Market Supply Chain

Initialisation Synopsis Due to our projection we need only consider how traders, agents and brokers initially, and in an ongoing way, come to know of one another. We omit details - "left as an exercise".

3.1.3 Discussion: Domain Requirements. We see a big difference in the two example cases: The The Citizen Smart Card case requires consideration of — as we shall later see — fairly, technologically detailed machine requirements, and in reality, as we shall see when all three kinds of requirements have been summarised, require quite an elaborate set of protocols. We are "close" to the hard technologies, and the protocol very much depends on hard technology dependability issues. The E-Business case is - most likely — to be implemented "on top" of Internet and the web; hence can rely on its underlying protocols; but requires, as we shall see, a mostly domain requirements-determined protocol. 


\subsection{Interface Requirements}

Interface requirements deal with "phenomena shared between users and the machine" (software + hardware). Interface requirements thus include consideration of such issues as graphical user interfaces (GUIs), input/output in general, and user/machine dialogue sequences.

3.2.1 The Citizen Smart Card. The interface requirements have, in this case, to do with the interface between the card and its user with respect to for example: (i) Authentication: For example sequence or simultaneity of pressing card with designated finger for its "print" recognition, uttering a certain phonetic sequence for "voice print" recognition, while aligning, say a left eye with a minute whole through the card, to see light trough it, for an "iris print". (ii) Transaction Processing: For example which card and terminal "buttons/icons" etc. to press/click in order to initiate, conduct and terminate update and query actions. EG.C. Since they only have marginal relevance to protocol engineering we omit further consideration.

3.2.2 E-Business. As for The Citizen Smart Card we omit detailed consideration of trader interfaces to the electronic market support. This is "classic" web-design!

3.2.3 Discussion: Interface Requirements. The The Citizen Smart Card case raises the most non-standard questions when it comes to interface requirements - it is so hard technology, so "gadget" biased. The E-Business case, in contrast, "falls" within the, by now, "classical" field of web and Internet design.

\subsection{Machine Requirements}

Machine requirements has to do with determining and describing such issues as (i) performance (temporal and spatial [response times, storage and other tangible resource consumption]), (ii) dependability (availability [up time], accessability [fairness], security [authentication, etc.], fault tolerance, etc.), (iii) maintainability (perfective, adaptive and corrective), (iv) portability (hardware and software platforms: development, execution and maintenance), (v) documentation, $\mathscr{E} c$.

3.3.1 The Citizen Smart Card. This case, of the two being treated, raises the most issues concerning machine, and in particular dependability requirements. They include: Availability: The up-time of the system of all smart card terminals. This issue has little to do 
with the concerns of protocol engineering - so we skip it. Accessibility: Provision - in the context of multiple users - of fair access to central resources. Since the entire idea of The Citizen Smart Card is "inverted" storage and computing the issue is almost mute. Anyway this issue also has little to do with the concerns of protocol engineering - so we skip it. Security: Ensuring protection of privacy of information and private ownership of securities instruments - in this case - leads to for example the triplet schema of eye iris, voice and finger print. There are some, however scant protocol issues related to this, but we skip them! Fault Tolerance: Provision of "soft" failures: That is, piecemeal, initially negligible degradation of service in case of lost or stolen cards and in case of terminal breakdowns. We have already, in the domain requirements section, had to presuppose a possibly elaborate scheme for protection against failures of these three kinds. They are the ones that makes, in our view, for an interesting set of protocols, three of which have been mentioned: The protocol(s) of "standard" (failure-free) operation, the protocol(s) for restoring lost or stolen cards, and the protocol(s) for routing around erroneous terminals. $\& c$.

3.3.2 E-Business. The availability, accessibility, and security requirements are assumed "taken care" of by an "underlying" Internet system. We focus just on the fault tolerance issue: If a trader "goes out of business", or electronically "breaks down", while many transactions, from and to many other traders are pending, then what? Here, for example, the possible simplicity of a supply chain protocol, as indicated earlier, is at stake. A proper protocol for handling this requires back-ups, duplication, "proxies" and the like. In other words: Protocol engineering "takes over"!

3.3.3 Discussion: Machine Requirements. We have seen that machine requirements could not be kept strictly separate from domain and interface requirements; and we have seen the diversity and differing intensity with which various machine requirements issues "flavours" protocol considerations. Finally we have seen that some protocol aspects, as dictated for example by machine requirements, can be taken care of by assumed implementation platforms, while others lead to additional protocols operating, as it were, "on top of platform protocols".

\subsection{Challenges}

There are two challenges. They can only be formulated now, after all aspects of the requirements have been listed: Domain, interface and machine requirements. 
3.4.1 Challenge I: The Citizen Smart Card Protocols. We challenge the reader to augment the The Citizen Smart Card synopses, and to informally and formally describe the protocols for handling (i) normal card/machine operation (insertion of forward and backward links and the copying of transaction data); (ii) re-establishment of a "new" card, when one is lost or stolen; and (iii) detection of and "rerouting" around failing machines.

3.4.2 Challenge II: E-Business Protocols. As for Challenge I we challenge the reader to informally and formally complete the EBusiness synopses, and in particular to informally and formally describe the implied E-Business protocols.

\subsection{Discussion: Requirements}

The various discussion sections, in connection with domain requirements, interface requirements, and machine requirements, seems to have exhausted the issue. Let us, however, just remind the reader that one example's "thin" domain description "explodes" into a hefty protocol requirements description, while another example's "substantial" domain description "fizzles" into a slightly augmented protocol requirements description, We refer to (one) The Citizen Smart Card versus (another) E-Business.

\section{FURTHER METHODOLOGY ISSUES \\ 4.1. Abstraction \& Modelling Principles and Techniques}

In general [1] outlines abstraction \& modelling principles and techniques applicable in any phase (domain, requirements and software design) of computing systems development: (a) property vs. model-oriented descriptions, (b) representation and operation abstraction, (c) denotations vs. computations (operational semantics), (d) hierarchies and compositions, (e) contexts, states and configurations, (f) applicative (functional), imperative, logic and parallel specification "programming", and many other specification principles and techniques. In this short paper we, unfortunately, must omit relating each and every one of these principles and techniques to protocol engineering as for example practised in this paper. 


\subsection{Domain Modelling Principles and Techniques}

$[1,2]$ outline a number of domain modelling principles and techniques: Modelling (i) static and dynamic, (ii) temporal and spatial, (iii) discrete, continuous and chaotic, and (iv) tangible and intangible attributes; modelling (v-vi) stake-holders and their perspectives; and modelling (vii) the intrinsics, (viii) the support technologies, (ix) the management \& organisation, (x) the rules \& regulations, and (xi) the human (correct, sloppy and criminal) behaviour facets.

Some relations were: (i) The The Citizen Smart Card PMR, PMJ, and Info information repositories were basically Static, whereas the Acc, Sec and Tick securities instrument (ie. money) repositories were Dynamic. (ii) There were Temporal relations between E-Business and The Citizen Smart Card transactions that determined crucial properties of protocols, and there we Spatial properties of the domain: Citizens gathering PMJ information "from far afield", and traders trading across geographical distance - all necessitating distribution of information and processing. (iii) The examples mostly exemplified Discrete systems. The fact that the stock market may behave Chaotically, or that electro cardiograms portray Continuous phenomena is of no interest for the systems such as we choose to see them. (iv) Most PMR information is Tangible: "Feelings" of being in bad health may be indicated by informal utterings in a PMR, but are Intangible. Whether to order or not to order a product, and in case of ordering, then from whom, may be Intangible attributes, but, as for the others, are of little concern to our protocol designs.

(v) We took a rather limited view of stake-holders, focusing only on patients, bank account holders, securities buyers and sellers, and commuters, respectively only on traders (consumers, retailers, wholesaler and producers). We did not consider health-care professionals, staff of financial institutions, transport company staff (drivers, etc.), nor the specific clerks, etc. of traders. (vi) And we took in account only a rather limited perspective. Enough to focus on the essentials for this article: The protocol development.

(vii) The citizen behaviour of Section 2.1.2 as well as the trader behaviour of Section 2.2.8 can be said to reflect the very basics, the Intrinsics of citizens and traders vis-a-vis the issues dealt with. (viii) We did not illustrate domain Support Technologies-prior to the advent of The Citizen Smart Card and E-Business. Once new requirements are expressed wrt. existing The Citizen Smart Card or E-Business system, then their card and terminal, respectively their assumed Internet technologies 
become support technologies. (ix) Past Management \& Organisation, past "bureaucracies" tended to prevent such "free" mechanisms as The Citizen Smart Card and E-Business. (x) The Rules \& Regulations of government supervised health-care, financial institutions and transport was not considered at all. Some such rules may affect protocols, however. (ix) We did consider Human Behaviour in both case examples - as they seem to influence, very strongly several aspects of protocol design: Loosing or stealing cards, issuing trading transactions "out of order" or forgetting to respond to such transactions. We refer to $[1,2]$.

\subsection{Requirements Modelling Principles and Techniques}

We have mentioned some requirements construction principles and techniques: Projection, instantiation, extension, and initialisation. They were illustrated in Section 2. We refer, in general to [1, 4].

\subsection{Software Design Principles and Techniques}

Domain and some, often all, interface requirements lead to software architecture design: A software architecture, to us, reflects exactly the issues with which the users are immediately and tangibly concerned. And these issues have to do with the domain, and with most of the interface. Machine requirements, and remaining interface requirements lead to program organisation design: They are intangible as far as the end-user is concerned, and may differ between computing and communication platforms. We refer to $[1,5]$.

\section{CONCLUSION}

This paper has - obviously - been written by a person who is not a researcher nor a practitioner of protocol engineering. Instead the author is a researcher and practitioner of the more "general" field of software engineering - if one can claim that such a broad field can be justified, let alone exists !

\subsection{What Have We Achieved ?}

What we have tried to do is to cast new light on protocol engineering. We claim, and we have shown, we believe, that it is useful - to the extent of being necessary — to first study and document the domain from which applications (also of protocols) emerge. We have indicated 
how protocols emerge from requirements, and how requirements emerge from domains.

\subsection{What Remains to be Done?}

The two examples were formulated as challenges. We have, ourselves, developed sketches and draft formalisations of some protocols, but would like to see others tackle the problem cases raised here !

\section{Acknowledgements}

The author gratefully acknowledges the kind invitation by Prof. Myungchul Kim (Inform. \& Comm. Univ., Korea), Dr. Byungmool Chin (Electronics Telecomm. Res. Inst., Korea), and Dr. Sungown Kang (Korea Telecom, Korea). Thanks to them, and Prof. Sam Chanson (HKUS\&T), I was challenged into formulating my thoughts on protocols and protocol engineering as emanating from domain and requirements engineering.

\section{References}

[1] Dines Bjørner. Software Engineering: Theory \& Practice. (Publisher is being contacted), 2002. This book represents the author's Chef d'Evres - the summary of more than 25 years of research, development and teaching.

[2] Annabelle McIver and Carrol Morgan, editors. Programming Methodology: Recent Work by Members of IFIP Working Group 2.3, chapter Dines Bjørner: "What is a Method ?" - A Study of Some Aspects of Software Engineering. IFIP WG2.3. MacMillan, Oxford, UK, 2001. To be published.

[3] The RAISE Language Group. The RAISE Specification Language. The BCS Practitioner Series. Prentice-Hall, Hemel Hampstead, England, 1992.

[4] Dines Bjørner. Domains as Prerequisites for Requirements and Software $6 c$. In M. Broy and B. Rumpe, editors, RTSE'97: Requirements Targeted Software and Systems Engineering, volume 1526 of Lecture Notes in Computer Science, pages 1-41. Springer-Verlag, Berlin Heidelberg, 1998.

[5] Dines Bjørner. Where do Software Architectures come from ? Systematic Development from Domains and Requirements. A Re-assessment of Software Engneering ? South African Journal of Computer Science, 1999. Editor: Chris Brink.

[6] Dines Bjørner. A Triptych Software Development Paradigm: Domain, Requirements and Software. Towards a Model Development of A Decision Support System for Sustainable Development. In ErnstRüdiger Olderog, editor, Festschrift to Hans Langmaack. University of Kiel, Germany, October 1999. 